Max Duperray*

\title{
Du fantastique littéraire: retour à la question du surnaturel chez James
}

DOI: http://dx.doi.org/10.12775/LC.2017.006

Résumé: L’article discute l'importance des phénomènes surnaturels dans les œuvres de Henry James. Dans son analyse, l'auteur fait référence aux vues critiques et théoriques de Virginia Woolf, Tzvetan Todorov et David Lodge. Il se réfère également aux œuvres d'écrivains tels que Nathaniel Hawthorne, Charles Dickens et Joseph Conrad.

Mots clés: surnaturel, fantastique, roman gothique, Henry James, Le Tour d'écrou

\section{Fantastyka literacka - problematyka zjawisk nadprzyrodzonych w twórczości Jamesa}

Streszczenie: Celem artykułu jest omówienie znaczenia zjawisk nadprzyrodzonych w twórczości Henry'ego Jamesa na podstawie tekstu Obrót śruby. W swojej analizie autor odwołuje się do wybranych tekstów krytyczno- i teoretycznoliterackich Virginii Woolf, Tzvetana Todorowa i Davida Lodge'a, a także do twórczości takich pisarzy, jak Nathaniel Hawthorne, Charles Dickens i Joseph Conrad.

Słowa kluczowe: zjawiska nadprzyrodzone, fantastyka, powieść gotycka, Henry James, Obrót śruby

\footnotetext{
* Professeur émérite à Aix-Marseille Université, spécialiste de la littérature gothique et fantastique, dans le domaine anglo-saxon, a publié sur le roman gothique anglais, sur Mary Shelley, De Quincey, Bram Stoker, ainsi que des études plus générales comme Londres, promenade sous un ciel couvert (2000), La folie et la méthode: la déréalisation en littérature (2001) ou La lame et la plume: une littérature de Jack l'Eventreur (2012). E-mail: maxduperray@gmail.com.
} 
ous ceux qui se sont intéressés au fantastique en littérature, dans le domaine anglosaxon en particulier, ont croisé Henry James sur leur chemin. C'est pour ma part, au tout début, le film de Clayton, The Innocents, l'adaptation brillante de «The Turn of the Screw» qui stimula ma curiosité et pour le fantastique et pour l'écrivain qui l'incarnait de façon si frappante. Maurice Lévy, le spécialiste français du roman gothique anglais, confiait volontiers qu'en la matière, il n'avait jamais lu quelque chose d'aussi fort. Or il avait passé une partie de sa vie académique à lire ces romans noirs du XVIIIe siècle qui avait fondé l'école frénétique et les codes de la peur dans les lettres de l'époque, une excursion dans le romantisme lugubre parfois lié à l'imaginaire germanique. Ce roman gothique était bien toujours présent dans le canevas de la «novella» de James, par quelque détour du langage, à savoir le souvenir des «Mystères d'Udolphe» de Mrs Radcliffe. Mais loin de s'être estompé dans une adaptation tardive, à une époque ultérieure qui avait surmonté tout penchant pour le surnaturel endiablé, il avait repris vigueur alors même que ses codes anciens n'avaient plus cours. On se souvient de la remarque de Virginia Woolf dans son essai sur le surnaturel en littérature: si l'on veut savoir ce que ressentaient nos ancêtres à la lecture des Mystères d'Udolphe rien de mieux que de lire Le Tour d'écrou ${ }^{1}$.

La transformation que le roman gothique avait subie, au sein d'un contexte de la finde-siècle très marquée par le goût du bizarre, mérite réflexion. Des rapports de James avec l'occulte, il n'en manque pas. Ne serait-ce qu'au sein du milieu familial où la fréquentation du paranormal avait été courante chez le père admirateur de Svedenborg ou le frère William, le psychologue. Très jeune encore il avait traduit la fameuse nouvelle de Prosper Mérimée, «La Venus d'Ille» qui lui fournit le canevas de sa propre histoire «The Last of the Valerii».

On trouve, chez ce grand représentant de l'école réaliste moderne, un élément hétérogène comme chez beaucoup de ses pairs, une part d'ombre ou bien aussi une échappatoire. Tous avaient exploré cet autre mode, Charles Dickens ou Thomas Hardy, Guy de Maupassant ou Honoré de Balzac, Robert Louis Stevenson ou Edith Wharton et bien d'autres, tous avaient fait ce 'détour', comme le disait un spécialiste du genre qui traqua ces textes marginaux des grands auteurs pas forcément associés au genre (Detours into the Macabre). Et de plus, il écrivit la biographie de Nathaniel Hawthorne et s'inscrit dans une généalogie des écrivains américains héritiers des Puritains très férus de sorcières et de fantômes. Il est aisé de retrouver dans ses écrits un patrimoine que Leon Edel a pris soin de réunir sous le simple titre d'histoires de fantômes - republié sous le titre «Le surnaturel», depuis «The Romance of Certain Old Clothes» de 1868 jusqu'à «The Jolly Corner» de 1908. De toute évidence James a une attirance pour ces histoires-là.

Ce qui, dans la critique, arrima davantage James au fantastique à proprement parler fut la parution en 1969 du livre de Tzvetan Todorov, Introduction à la littérature fantastique, plus clairement énoncé dans la traduction anglaise ultérieure comme A Structural Approach to a Literary Genre. Avec cet essai un tournant s'opérait dans l'appréciation des textes qu'on avait jusqu'alors reconnus à quelque choix thématique significatif.

La thèse soutenue était celle d'une hésitation herméneutique du lecteur entre deux interprétations des phénomènes décrits: celle de l'esprit rationnel et celle de l’adhésion à la

1 V.Woolf, «The Supernatural in Fiction», The Complete Collection, Amazon Digital Services LLC 2016, p. dccxlvi. 
surnature. Pour mériter l'étiquette générique le texte devait faire perdurer jusqu'à son terme cette double possibilité sans que jamais on ne puisse trancher entre l'une et l'autre. Todorov avait le grand mérite de proposer une méthode à reconnaître la validité d'une étiquette. Il n'en avait pas moins rencontré quelque difficulté à classer les productions littéraires selon un schéma aussi rigoureux, à tel point qu'il avait dû se résoudre à introduire des sous-catégories, essentiellement celle de l'étrange face à celle du merveilleux qui s'alliaient souvent, en quelque hybridation féconde, avec le tronc commun du fantastique.

Or parmi les textes qu'il avait trouvé comme illustration de sa théorie, très rares au demeurant, il y en avait un qui lui servait de référence incontournable et c'était The Turn of the Screw de James, là où, jusqu'au bout, l'impossibilité perdurait à se décider sur la nature des événements rapportés. Ainsi passait-on d'une lecture thématique avec tout ce que la nouvelle mettait en scène - le château, la gouvernante esseulée, l'intrusion des fantômes, la peur du mal qui rôde sans s'avouer -bref ce que l'auteur désignait lui-même comme une histoire à faire peur que l'on raconte au coin du feu - à une autre qui s'intéressait au mécanisme du récit. Il s'agissait de maintenir en haleine un lecteur en lui faisant toucher du doigt l'ambiguiité de la vérité. Comme Stevenson le rappelle dans A Humble Remonstrance, James avait toujours plaidé le caractère sacré de la vérité aux yeux du romancier, vérité dérangeante d'autant plus qu'on échouait à la désigner. En tout cas dans cette approche s'affirmait la primauté de la forme sur la matière au profit d'une définition inaliénable d'un genre structurellement défini.

Si Le Tour d'écrou s'érigeait en modèle théorique, il n'est pourtant pas sûr que la théorie en épuisait la nature. Todorov traitait du Tour d'écrou comme un exemple prélevé de son contexte. Il était légitime qu'il le fasse pour la cohérence de sa démarche. Cependant il se privait d'une mise en écho de la nouvelle avec toute l'œuvre qui aide à en cerner la vraie nature. Il réduisait aussi la richesse infinie d'un genre en voulant assimiler son fonctionnement à un archétype par définition universalisant. Une critique de sa lecture structuraliste a été faite dans une thèse sur le spectral chez James ${ }^{2}$ qui avance que Todorov lisait James à la lumière de la poétique du récit gothique qui est un modèle pré-jamesien. Selon lui il s'agissait d'un schéma statique à l'opposé du mouvement rotatoire du récit, celui qui s'ancre dans le concept de tournant («turn»). - Le critique ne tenait pas compte, cependant, d'un autre essai paru à la même époque.

Car avec «Les Fantômes de James» (1969), dans Poétique de la Prose, Todorov replace bel et bien la nouvelle dans l'ensemble des récits de James, histoires de fantômes au moins, pour en évaluer la spécificité. Il remarque en passant que, lorsque les lecteurs choisissent les récits qui pourraient entrer dans l'anthologie d'Edel, ils n'arrivent jamais au même résultat. Todorov se retrouve, en creusant la problématique de ces récits, devant une difficulté rencontrée dès la rédaction de son traité structuraliste: sa théorie a du mal à cadrer avec le texte moderne et à réduire la modernité du texte jamesien. Le statut de 'fantastique' y est souvent remis en cause, par subordination du surnaturel à autre chose, c'est-à-dire au merveilleux, à un psychologisme, à l'allégorie par exemple, au détriment de ce que Todorov appelle le genre. Il avoue que chez James le fantastique n'a aucune homogénéité et il renonce à chercher une adéquation. Plutôt «se tourner vers le dessein qui unit les textes de James $»^{3}$, c'est-

\footnotetext{
2 T. J. Lustig, Henry James and the Ghostly, Cambridge 1993.

3 T. Todorov, «Les Fantômes de James» (1969), Poétique de la prose, Paris 1971, p. 191.
} 
à-dire un choix qu'il qualifie de «thématique»: James préfère «la perception à l'action, la relation à l'objet à l'objet lui-même, la temporalité circulaire au temps linéaire, la répétition à la différence» ${ }^{4}$. C'est une correction importante à sa glose sur le genre. Pourtant il ne revient pas au Tour d'écrou qui, de ce fait, est maintenu dans son statut de texte-phare du fantastique littéraire.

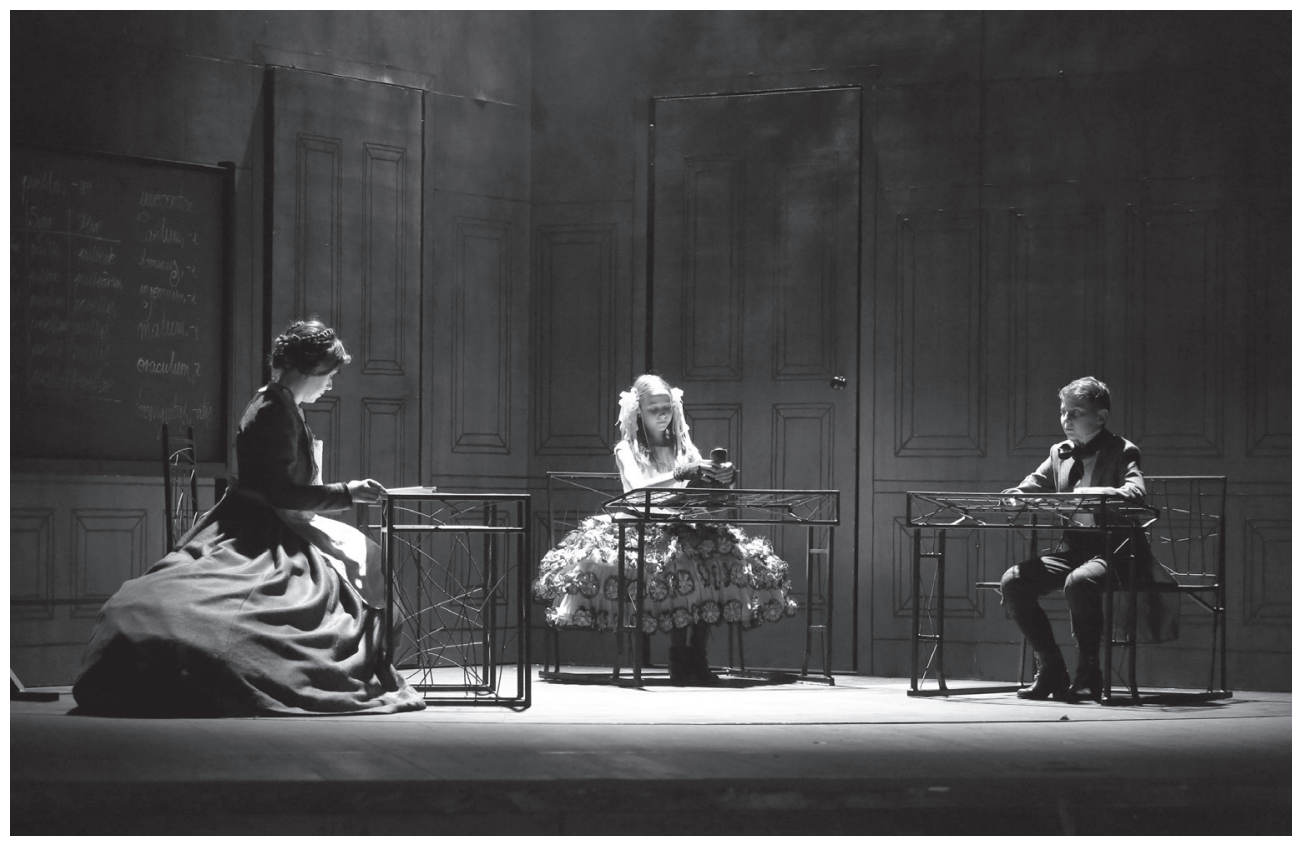

Le tour d'écrou. Photo par Marek Grotowski

David Lodge l'avait dit: le genre stricto sensu a un statut monologique et il n'y a jamais dans les grandes œuvres qu'une présence oblique du genre ${ }^{5}$. D'aucuns ont même souligné que l'inquiétant est opposé au suspense (qu'implique l'hésitation herméneutique) car le postulat de la surnature doit être reconnu comme une image d'un réel, entendu sans doute dans une acception lacanienne. Sans vouloir s'impliquer dans un discours théorique quelconque, on remarquera que ces approches rendent compte assez bien du danger qu'il y a à vouloir poser un canevas préétabli à une écriture qui, par définition, s'émancipe de toute formule attendue. En tout cas cette présence oblique que Lodge avait indiquée est cela même que James dénomme «indirection». Le fantastique serait donc ici un détour. D'où la vertu de l'anamorphose qu'illustre son grand roman The Ambassadors. Mais ce détour est plus complexe que la simple mise au service d'une cause, qu'elle soit idéologique ou morale.

La notion même de fantastique serait-elle plus embarrassante qu'utile? Le terme n'est pas courant en anglais. Rosemary Jackson dans son approche d'une «littérature de la subversion» use du vocable «Fantasy» par exemple. La terminologie n'est pas vaine: elle reflète des façons de voir. Un certain cartésianisme latin épris de classifications définit plus étroitement le «fantastique». James a quelquefois semblé tenir le genre en peu d'estime.

\footnotetext{
4 Ibidem, p. 192.

5 D. Lodge, After Bakhtin: Essays on Fiction and Criticism, New York 1990, p. 66.
} 
On se souvient de la façon dont il dévalorise Le Tour d'écrou en le faisant passer pour une «amusette», ou «un jeu d'esprit» - en français dans le texte -. Le fantastique est un merveilleux, une autre forme du conte de fées, selon ses propres mots. Finalement le genre sert le réalisme quand celui-là a besoin d'un rehausseur, comme un visage d'un rimel. Woolf l'avait dit à propos du surnaturel: « $[\ldots]$ in our twilight state it is better to guess than to assert, to feel than to classify our feelings.» Elle s'orientera donc vers une phénoménologie: le surnaturel chez James n'est pas une répétition du gothique, mais un moment crucial du texte, une harmonie suprême quoique choquante ${ }^{6}$.

Il n'empêche que la relation de James avec un genre qui perdure et se diversifie dans le temps, demeure une question à poser. D’autant que le déni implicite du romancier n'est pas à prendre au pied de la lettre. Il est, comme toute assertion de sa plume, fondamentalement ambigu. Et cela n'est pas dépourvu d'une ironie discrète qui habite souvent le devenir des récits comme dans tous les cas où le scepticisme légitime envers les fantômes reçoit une réponse cinglante et inattendue, où le faux fantôme appelle la visite des vrais («The Ghostly Rental» par exemple).

On a répondu aussi à cette question sur la fonction ou la spécificité du surnaturel par des références littéraires en élargissant le contexte à une époque. Rosemary Jackson ainsi aborde la notion de «fantastic realism», une hybridation notoire, et reprend, avec la thématique du double, la triade George Eliot (The Lifted Veil), Joseph Conrad (The Secret Sharer ou Heart of Darkness) et Henry James («The Jolly Corner»). Cela impliquerait qu' il y a au moins une rencontre entre l'auteur et une manière d'écrire le fantastique qui serait propre à son temps. Il est vrai que Conrad est le plus proche d'une variante du surnaturel qui est celle de James: un vide plutôt qu'un plein, une abstinence plutôt qu'un engagement. Au «cœur des ténèbres» la question est sans réponse et le parcours sans aboutissement, mais l'ultime vision est potentiellement dérangeante.

D'autre part la façon dont l'auteur esquive la question, en donnant à sa meilleure nouvelle une place ancillaire ou une fonction ludique, ne diminue pas l'importance de la tradition gothique. Pour être tenu à distance le fantastique n'en est pas moins troublant dans sa présence discrète ou reniée. Si l'on revient aux textes du XIXe de la trilogie de Jackson, il faut bien admettre que, loin d'alléger l'inquiétude qu'ils génèrent, ils la dramatisent dans leur non-réponse. Woolf souligne la beauté d'un surnaturel apaisé, serein, comme en musique l'accord final du morceau, mais elle emploie aussi le mot terrible d'obscène pour désigner ce que cet accord fait entendre aussi «The supernatural is brought in to provide $[\ldots]$ the queerest of shocks - tranquil, beautiful, like the closing of chords in harmony; and yet somehow obscene ${ }^{7}$. Voilà qui rappelle le coup final de Heart of Darkness «The horror, the horror!», une réalité odieuse dans son imprécision. L'indétermination laisse le champ libre à la réception du lecteur: une absence fondamentale de référence ou de présence incontournable, des antonymes qui s'annulent. Les histoires qui convoquent le surnaturel ne sont pas précisément des histoires de fantômes, mais des souvenirs d'histoires de ce type. James appelle souvent ces textes-là des «romances $»$ comme $\ll$ De Grey». C'est un retour au texte fondateur du gothique précisément situé déjà entre «novel» et «romance». Le surnaturel en tant que tel n'est pas toujours explicite; des œuvres comme «Professor Fargo»,

\footnotetext{
6 V. Woolf, «The Supernatural in Fiction», p. dccxlvi.

7 Ibidem, p. dccliii.
} 
l'histoire diabolique de l'homme roux ou The Portrait of a Lady en témoignent. Edel parle de $\ll$ Ghostly Tales» et non de «ghost stories $»$. L'accent est donc moins sur le sujet que sur la façon de la raconter. Le retour sur un passé aussi bien dans une biographie que dans une manière d'écrire, l'usage des tropes du gothique, tout se lit comme un souvenir tenace à revisiter James, «un hôte résigné et ironique de l'enfer» écrit Jorge Luis Borges ${ }^{8}$.

La quête infructueuse d'une critique qui a épuisé les textes et donné plus d'un tour à la vis de l'écrou, pour en saisir le cœur ou le sens, trouve son illustration dans le célèbre «Figure in the Carpet» où le secret relatif à l'expression artistique s'hérite de personnage en personnage et les fait disparaître l'un après l'autre. A suivre brièvement une chronologie des lectures du texte tant discuté du Tour d'écrou, on verra bien les étapes d'une usure qui épuise les pistes possibles d'une interprétation du sens pour parvenir à une exploration de sa textualité même, soit dans la direction d'une structure à l'œuvre, soit dans celle d'un inconscient ou des deux.

Le retour à une forme quelque peu dépassée, l'histoire de fantôme à la manière des histoires de Noël de Dickens, se superpose à l'obsession du passé qui se lit partout chez James. D'où cet aboutissement ultime à une histoire de fantôme (The Sense of the Past) qui ne dit pas son nom, mais se cristallise sur le retour sur soi dans le cadre d'un récit historiographique - comme sur le fantôme de l'homme qu'on a été ou aurait pu être. Cela se construit dans le retour du voyageur dans la demeure new-yorkaise («The Jolly Corner») ou dans l'esquisse d'un voyage dans le temps (The Sense of the Past), sans doute un vain espoir de s'affranchir du temporel. Mais cela contribue à une présence fantomatique de l'un et l'autre, l'ego et son alter ego. Ce statut fantomatique irradie la scène de façon particulièrement frappante dans «A Passionate Pilgrim» où l'Angleterre revisitée par le protagoniste pour recouvrer un avoir usurpé assume le rôle du rêve d'une architecture enivrante dans un écrin de verdure inviolée.

Il est remarquable dans tous ces cas que la textualité, texte et écriture, constitue une ligne conductrice. On l'a déjà dit à propos du Tour d'écrou, la gouvernante est d'abord une lectrice. Elle voit les fantômes, a-t-on pu dire, comme les lettres de la page de son livre (Gert Buelens). Dans «A Passionate Pilgrim» c'est à Oxford, la cité de l'esprit et de l'écriture muséifiés, que l'histoire se situe.

Un des tropes fondamentaux du Tour d'écrou est l'écrit dénié: la gouvernante est interdite d'écriture et ne doit pas informer le maître de ce qui se passe à Bly. C'est un trope très courant dans le roman gothique, celui qui fonde Udolpho où l'héroïne ne peut lire les textes que son père lui a légués. En rapprochant James à Maupassant, un lecteur compare «Apparition» du romancier français au Tour d'écrou précisément sur cette question. Le protagoniste a mission d'aller chercher secrètement des papiers qu'il ne doit pas lire. Le critique note le retour, dans le nouvelle de James, d'un trope qui existait déjà dans «Apparition» de Maupassant: deux textes qui s'articulent sur «un échange complexe de lettres tenues sous clé»?.

Cette ambiguité qui mine le texte en lui refusant une légitimité le pare d'un statut fantomatique. On a pu, comme Shoshana Felman en particulier ${ }^{10}$, faire ressortir le recoupe-

\footnotetext{
8 J. L. Borges, La préface à l'Humiliation des Northmore, Livre des préfaces, Paris 1980.

9 B. Terramorsi, «Maupassant et James: les tours du fantastique» Europe Août-Septembre (1983), p. 138.

10 S. Felman, Writing and Madness, Palo Alto 2003, p. 168.
} 
ment de la forme perdue - «a lost form» - et le récit qui s'éloigne du lecteur par la multiplication des narrateurs relais. D'où une contamination entre un texte sans origine et une maison sans maître et donc hantée.

Le surnaturel devient dans ces textes une sorte de révélateur ironique. De nombreuses nouvelles sont emblématiques de ce procès. Dans «The Friends of the Friends» l'homme et la femme réunis par une tierce personne pour leur aptitude à voir des fantômes passent leur existence à rater leur rencontre et ne la réussissent qu'après la mort de l'héroïne pour le meilleur et le pire.

Il faut donc revoir l'évaluation du surnaturel dans l'œuvre de James. D'aucuns ont relevé chez lui le recours à une terminologie qui renvoie au fantastique (Valérie Tritter par exemple). Dans les Carnets, à la date du 9 août 1900, James s'attarde sur les difficultés de la composition de The Sense of the Past. Il cherchait quelque chose qui serait aussi simple que «The Turn of the Screw», mais moins direct, si l'on peut dire: «less grossly and merely apparitional» ${ }^{11}$, une formule qu'il énonce par le terme 'fantasticated': «the fantasticated is, for this job, my probable formula». Il poursuit en s'arrêtant précisément sur la question du genre: «And I know what I mean by it, as differentiated from the type, the squeezed sponge of The Turn of the Srew. 'Terror' peut bien en être and all the effective malaise $>^{12}$.

En cela Todorov était fondé à distinguer The Turn of the Screw pour le placer au panthéon du fantastique plus classique.

Ce qui intéresse en effet le romancier c'est de savoir comment dépasser le fantastique de la «novella» pour aller vers un processus qui ferait comprendre le néologisme de «fantasticate $\gg$ : «I was rather taken with Howell's suggestion of an 'international ghost' $\gg-$ entendons sans doute ce qu'il développe ensuite dans les rencontres de l'Américain voyageant dans le temps européen, selon la formule plus ramassée de «The Passionate Pilgrim» ou, de façon plus intellectualisée dans «The Jolly Corner». Il va avancer dans le sens d'un dialogisme avec l'altérité au sein de la même personne. Cela ne signifie pas qu'il ait relégué le genre à une formule primaire. Son assimilation du fantastique à un merveilleux ne doit pas être lue comme une dévalorisation. Si l'on se reporte à ce qu'il écrit dans la préface à «The Altar of the Dead», il revient à quelques histoires réunies: «The Friends of the Friends», «Owen Wingrave», «Sir Edmund Orme», «The Real Right Thing». Il convient qu'il y a là quelque chose qui touche la sensibilité et qu'il appelle «the fairy-tale side of life», une forme hybride de primarité et d'émerveillement. La forme la plus accomplie du conte de fées est «the ghost story»: «It may seem odd $[\ldots]$ in a search for the amusing $[\ldots]$ to try to steer wide of the silly by hugging close to the supernatural». James confère au surnaturel une place de choix. Il est, écrit-il, l'épaisseur, cet effet de loupe que prend l'histoire de la relation normale du sujet avec les choses ${ }^{13}$. La poétique de Todorov n'est donc pas hors sujet, même si l'hésitation herméneutique n'est pas ici la règle.

Borges avouait: «j’ai travaillé sur une anthologie encyclopédique de la littérature fantastique, j'ai traduit Kafka, Melville et Bloy; je ne connais pas d'œuvre plus étrange que celle d'Henry James». Borges traitait volontiers les écrivains de son anthologie de «professionnels de l'irréel», mais James «[est] un hôte résigné et ironique de l'Enfer». Il procède par

11 H. James, The Notebooks of Henry James, F. O. Mathiessen (éd.) et Kenneth E. Murdock (éd.), New York 1955, p. 299.

12 Ibidem, p. 300.

13 Prefaces to the New York Editions, 1984, p. 1259. 
«omission d'une partie du roman» et ce que l'on prend pour un mélodrame est en fait une série «d'hyperboles ou d'emphases dans la trame du récit» ${ }^{14}$.

Dans ce sens James apporte sa pierre à l'émergence d'un fantastique littéraire, mental et scriptural, spéculaire, situé dans cette zone liminale entre l'événementiel et son ombre.

\section{Bibliographie}

Borges, Jorge Luis, Livre des préfaces (suivi de) Essai d'autobiographie, trad. Françoise-Marie Rosset, Paris: Gallimard, 1980.

Buelens, Gert, «The Sense of the Past, History and Historical Criticism», Palgrave Advances in Henry James Studies, Londres: Palgrave Macmillan, 2007.

Duperray, Max, « Du Spectral, possession et exorcisme: les histoires de fantômes d'Henry James comme mélodrame mental», La Folie et la méthode, Essai sur la déréalisation en littérature, Paris: l'Harmattan, 2001.

—, «L'inquiétant et le texte sans propriétaire», L'inquiétant, Psychanalyse et recherches universitaires (PERU), Rennes: Presses Universitaires de Rennes, 2003.

Felman, Shoshana, Writing and Madness (Literature, Philosophy, Psychoanalysis), Palo Alto: Stanford University Press, 2003.

Haining, Peter, éd., Detours into the Macabre, Londres et Sydney: Pan Books, 1972.

Jackson, Rosemary, Fantasy, The Literature of Subversion, Londres et New York: Methuen, 1981.

James, Henry, The Notebooks of Henry James, F. O. Mathiessen (éd.) et Kenneth E. Murdock (éd.), New York: George Braziller, Inc., 1955.

—, The Complete Tales of Henry James, Leon Edel éd., Londres: Rupert Hart-Davis, 1962.

—, «Preface to 'The Altar of the Dead'», Prefaces to the New York Editions, New York: The Library of America, vol. II, 1984.

Lodge, David, After Bakhtin: Essays on Fiction and Criticism, New York: Routledge, 1990.

Lustig, T. J., Henry James and the Ghostly, Cambridge: Cambridge University Press, 1993.

Terramosi, Bernard, «Maupassant et James: les tours du fantastique» Europe Août-Septembre, 1983.

Todorov, Tzvetan, Introduction à la littérature fantastique, Paris: Seuil, 1969.

—, «Les fantômes de Henry James» [1969], Poétique de la prose, Paris: Poétique / Seuil, 1971.

Tritter, Valérie, «Henry James», Encyclopédie du fantastique, Paris: Ellipses, 2010.

Woolf, Virginia, «The Supernatural in Fiction», Virginia Woolf: The Complete Collection. Amazon Digital Services LLC, 2016 [Times Literary Supplement, Jan, 31, 1918 as Across the Border].

—, «Henry James’s Ghost Stories», Times Literary Supplement, December 22, 1921.

14 J. L. Borges, La préface à l'Humiliation des Northmore, 1980. 